\section{Hiperpotasemia extrema no fatal en un caso de acidosis láctica por sobreingesta de metformina}

\author{
RIENZI DÍAZ ${ }^{1,2,3}$, JORGE VEGA ${ }^{1,4,5}$, HELMUTH GOECKE ${ }^{1,4}$
}

\section{Non-fatal hyperkalemia in lactic acidosis due to metformin overdose. Report of one case}

We report a 74-year-old man with diabetes mellitus type 2 and hypertension, who recently underwent coronary bypass surgery due to severe triple vessel disease receiving cardiological and combined antidiabetic therapy, including metformin $4 \mathrm{~g} /$ day. He was admitted with abdominal pain, nausea, vomiting, diarrhea and loss of consciousness. At admission, he was disoriented and agitated with signs of poor perfusion. His blood pressure was $80 / 70 \mathrm{mmHg}$, pulse rate 40 beats $/ \mathrm{min}$, respiratory rate 20-breaths/min, and axillary temperature $35^{\circ} \mathrm{C}$. Biochemical profile revealed an extreme hyperkalemia of $15.4 \mathrm{mEq} / \mathrm{L}$ (double checked), elevated creatinine, uremia and brain natriuretic peptide; hypoglycemia (blood glucose $68 \mathrm{mg} / \mathrm{dl}$ ) and normal C Reactive Protein. Arterial blood gases revealed severe lactic acidemia. The electrocardiogram showed sinus bradycardia, simple AV block, widened QRS with prominent T wave and prolonged QT. He was admitted to the Intensive Care Unit (ICU) with the suspicion of lactic acidosis associated with metformin, receiving fluid management, intravenous hypertonic glucose plus insulin and sodium bicarbonate, mechanical ventilation, vasopressor therapy, a temporary pacemaker lead, in addition to continuous venovenous hemodiafiltration. Two days later, the patient experienced a significant clinical improvement with normalization of the acid-base status, plasma lactate and potassium levels. On day 9, diuresis was recovered, creatinine and uremia returned to normal levels and the patient was discharged from the ICU.

(Rev Med Chile 2015; 143: 391-395)

Key words: Acidosis, lactic; Hyperkalemia; Metformin.

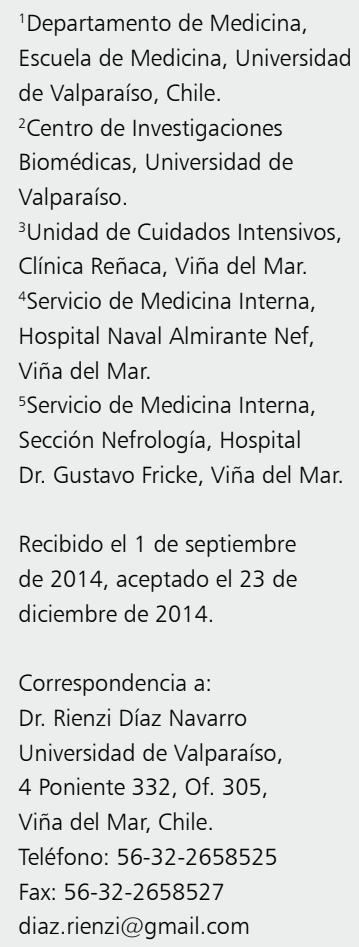

E n la mayoría de los pacientes portadores de diabetes mellitus tipo 2 (DM2), el tratamiento farmacológico incluye más de un fármaco ${ }^{1}$. Entre estos, la metformina, una biguanida, es considerada un medicamento estándar. Es de primera línea en el tratamiento de esta enfermedad metabólica, producto de su eficacia, seguridad y reducción de las complicaciones cardiovasculares ${ }^{2-4}$, tiene una absorción gastrointestinal rápida y se elimina por filtración glomerular y secreción tubular.

Una complicación latente con el uso de bi- guanidas es la acidosis láctica, cuya asociación fue ampliamente demostrada con fenformina y buformina ${ }^{5-8}$, determinando que ambos fármacos fueran retirados del mercado. Por otra parte, aunque algunos estudios muestran que el uso de metformina no estaría asociada a la generación de acidosis láctica9 ${ }^{9-11}$, se ha demostrado que es una complicación de baja incidencia pero elevada mortalidad $^{12-17}$. Los factores predisponentes incluyen la insuficiencia renal y hepática, el alcoholismo y las patologías asociadas con hipoxia (insuficiencia cardiaca, enfermedad pulmonar, cirugía), la edad 
avanzada, deshidratación, sepsis y la sobredosis 9 . $\mathrm{Su}$ incidencia alcanzaría a 9 casos por 100.000 pacientes, distinto a los 40-64 casos por 100.000 de las otras biguanidas ${ }^{18}$, y la mortalidad puede alcanzar hasta $50 \%{ }^{16,19}$.

Comunicamos el caso de un paciente con hiperpotasemia extrema asociada a una acidosis láctica severa, muy probablemente desencadenada por una sobredosis de metformina, exitosamente tratado con hemodiafiltración veno-venosa continua (HDFVVC).

\section{Caso clínico}

Hombre de 74 años de edad, con antecedentes de DM2, hipertensión y cirugía de revascularización coronaria reciente por enfermedad coronaria severa de tres vasos, diagnosticada después de un evento coronario agudo. Presentaba disfunción ventricular izquierda con fracción de eyección $40 \%$ y disfunción renal con creatininemia de 1,49 $\mathrm{mg} / \mathrm{dL}$. Dos semanas después del alta hospitalaria, fue ingresado a la Unidad de Cuidados Intensivos con dolor abdominal, náuseas, vómitos y lipotimia. La familia refería que además del tratamiento cardiológico (bisoprolol 1,25 mg/día, aspirina $100 \mathrm{mg} /$ día; atorvastatina $40 \mathrm{mg} /$ día; furosemida $20 \mathrm{mg}$ cada $12 \mathrm{~h}$; espironolactona $25 \mathrm{mg}$ /día), el paciente estaba tomando metformina $1.000 \mathrm{mg}$ cada 12 h y 1 tableta cada 12 h de una combinación de vildagliptina $50 \mathrm{mg}+$ metformina 1.000 $\mathrm{mg}$ ), vale decir, $4.000 \mathrm{mg}$ de metformina al día. Al ingreso, el paciente estaba desorientado y agitado con signos de mala perfusión, presión arterial de $68 / 38 \mathrm{mmHg}$, frecuencia cardiaca de 40 latidos/ min, frecuencia respiratoria de 20 respiraciones/ min y temperatura $35^{\circ} \mathrm{C}$. En el laboratorio destacaba una hiperpotasemia extrema de $15,4 \mathrm{mEq} / \mathrm{L}$ (doble verificación); sodio $133 \mathrm{mEq} / \mathrm{L}$; cloro 118 $\mathrm{mEq} / \mathrm{L}$; creatininemia $5,16 \mathrm{mg} / \mathrm{dL}$, uremia 115,6 $\mathrm{mg} / \mathrm{dL}$; glicemia $68 \mathrm{mg} / \mathrm{dl}$; PCR 3,7 mg/L; CKtotal $15 \mathrm{U} / \mathrm{L}, \mathrm{CK}-\mathrm{MB} 1,3 \mathrm{U} / \mathrm{L}$; troponina $<0,03$ ng/L; y péptido natriurético cerebral $1.140 \mathrm{pg} / \mathrm{mL}$. Gases arteriales 1 (oxígeno $\mathrm{O}_{2} 3 \mathrm{~L} / \mathrm{min}$ ): acidosis metabólica: $\mathrm{pH} 7,08 ; \mathrm{PCO}_{2} 22,2 \mathrm{mmHg} ; \mathrm{PO}_{2}<60$ $\mathrm{mmHg}$, bicarbonato $6,4 \mathrm{mEq} / \mathrm{L}, \mathrm{EB}-23,7$ y lactato $51,7 \mathrm{mEq} / \mathrm{L}$. Electrocardiograma: bradicardia sinusal, bloqueo AV simple, QRS ensanchado con onda $\mathrm{T}$ prominente y QT prolongado 457 ms (QTc $463 \mathrm{~ms}$ ) (Figura 1). La terapia incluyó la administración de glucosa hipertónica más insulina, ventilación mecánica, drogas vasoactivas y hemodiafiltración veno-venosa continua. Dos días después, el paciente experimentó una mejoría clínica significativa con normalización del estado ácido-base y del potasio plasmático, además de mejoría de otros parámetros de laboratorio, (Tabla 1). Al noveno día, el paciente recuperó la diuresis y fue dado de alta de la Unidad de Cuidados

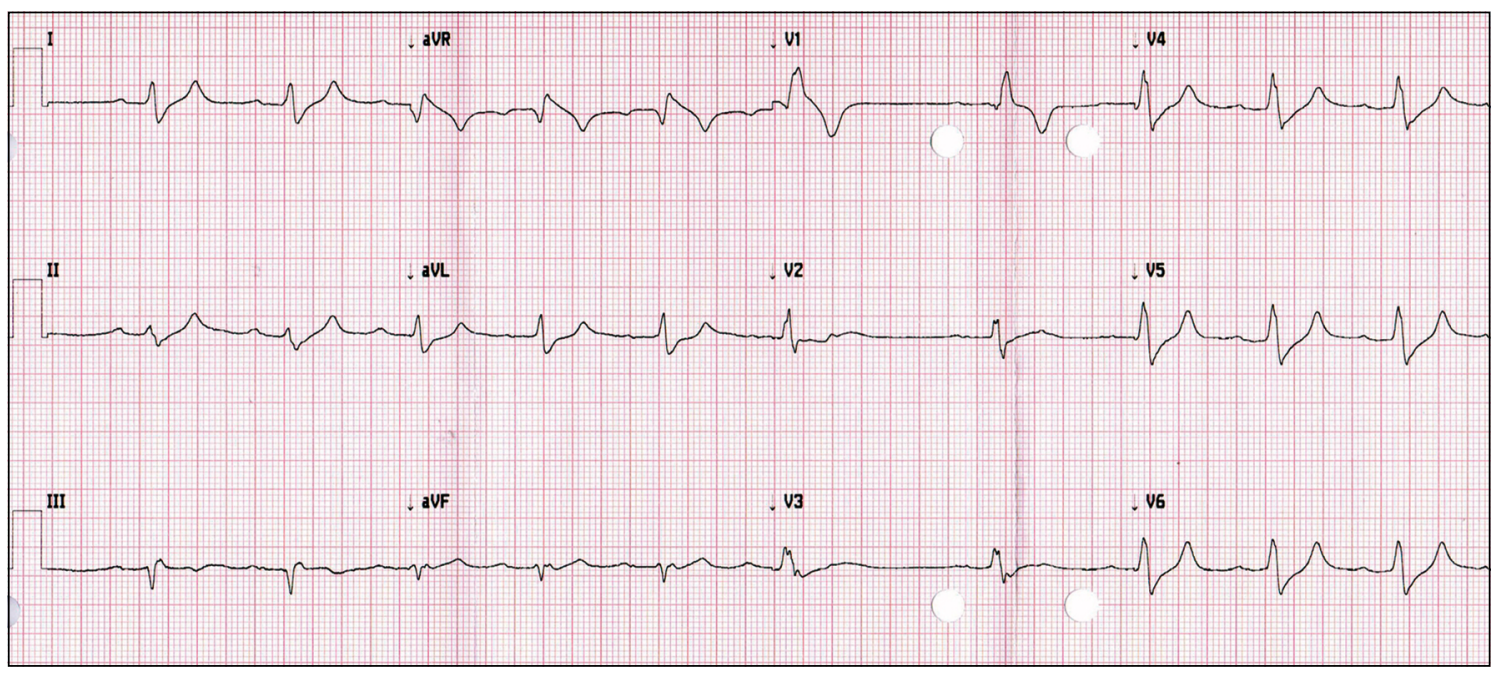

Figura 1. Electrocardiograma muestra bradicardia sinusal, bloqueo AV simple, QRS ensanchado con onda T prominente y QT prolongado [457 ms/QTc $463 \mathrm{~ms}$ ). QTc, QT corregido. 
Tabla 1. Exámenes de laboratorio

\begin{tabular}{|lccc|}
\hline & 2 semanas antes del ingreso & Ingreso & 2 días post tratamiento \\
$\mathrm{Na}(\mathrm{mEq} / \mathrm{L})$ & 131 & 133 & 135 \\
$\mathrm{~K}(\mathrm{mEq} / \mathrm{L})$ & 4,2 & 15,4 & 3,3 \\
$\mathrm{Cl}(\mathrm{mEq} / \mathrm{L})$ & 94 & 118 & 98 \\
Ácido Láctico $(\mathrm{mEq} / \mathrm{L})$ & - & 51,7 & 21,3 \\
$\mathrm{pH}$ & - & 7,08 & 7,43 \\
$\mathrm{PCO}_{2}(\mathrm{mmHg})$ & - & 22,2 & 35,5 \\
$\mathrm{PO}_{2}(\mathrm{mmHg})$ & - & $<60$ & 90,9 \\
$\mathrm{Bicarbonato}(\mathrm{mEq} / \mathrm{L})$ & - & 6,4 & 24,3 \\
$\mathrm{BE}(\mathrm{mEq} / \mathrm{L})$ & - & $-23,7$ & 0,3 \\
$\mathrm{Saturación} \mathrm{O}_{2}(\%)$ & - & $<60$ & 97,3 \\
$\mathrm{CO} \mathrm{z}_{2}$ total $(\mathrm{mmHg})$ & - & 7,1 & 25,4 \\
Creatininemia $(\mathrm{mg} / \mathrm{dL})$ & 1,27 & 5,16 & 1,68 \\
Uremia $(\mathrm{mg} / \mathrm{dL})$ & 32,1 & 115,6 & 38,5 \\
\hline
\end{tabular}

Intensivos. A los 15 días de evolución, el paciente está asintomático con volumen urinario $>1.500$ $\mathrm{ml} /$ día, creatininemia de $1,08 \mathrm{mg} / \mathrm{dL}$ y uremia de $32,1 \mathrm{md} / \mathrm{dL}$.

\section{Discusión}

La acidosis láctica asociada al uso de biguanidas (fenformina, buformina) está ampliamente demostrada $^{5-8}$. La acidosis asociada al uso de metformina, es de baja incidencia pero elevada mortalidad $^{16}$. Esta asociación es controvertida; sin embargo, el criterio diagnóstico está basado en la combinación de acidosis metabólica con anión GAP alto, ácido láctico aumentado, en pacientes que reciben metformina y desarrollan un cuadro clínico de dolor abdominal, trastorno del nivel de conciencia y taquipnea, sintomatología que presentó nuestro paciente ${ }^{16}$.

La acidosis láctica por metformina y otras biguanidas, es producto del aumento de la glucólisis anaerobia, inhibición de la gluconeogénesis hepática y al aumento de la producción intestinal de lactato, siendo la disfunción renal el factor predisponente más importante al dificultar su eliminación favoreciendo su acumulación.

En nuestro paciente, la acidosis con alta pro- babilidad resultó de la sobredosis de metformina ingerida por el paciente, $4.000 \mathrm{mg} /$ día [dosis máxima recomendada $2.550 \mathrm{mg} / \mathrm{día}^{19}$, asociada a una agravación de la disfunción renal preexistente por deshidratación (secundaria a vómitos, diarrea y falta de ingesta), así como al bajo débito cardiaco por disfunción ventricular, esto explicaría la marcada elevación del BNP.

La observación más dramática en este caso fue la presencia de una hiperpotasemia extrema de $15,4 \mathrm{mEq} / \mathrm{L}$, acompañada de bradiarritmia severa que requirió del uso de una sonda marcapasos transitoria, administración de glucosa hipertónica y rápida corrección de la acidosis metabólica con bicarbonato intravenoso e inicio de HDFVVC ${ }^{12,14,20,21}$. La hiperpotasemia es una condición potencialmente fatal, definida por un nivel sérico de $\mathrm{K}>5,5 \mathrm{mEq} / \mathrm{L}$, mientras que niveles sobre $7 \mathrm{mEq} / \mathrm{L}$ representan hiperpotasemia severa $^{22}$.

La acidosis metabólica aguda produce el movimiento del $\mathrm{K}$ desde el espacio intracelular al extracelular, mediante la utilización de numerosas vías de transporte de iones entre las células musculares y el espacio extracelular ${ }^{23}$. La concentración plasmática de potasio se incrementa en $0,6 \mathrm{mEq} / \mathrm{L}$ por cada reducción de 0,1 unidades del $\mathrm{pH}$ extracelular ${ }^{24}$. 
Este paciente recibía varios medicamentos para el tratamiento de su cardiopatía coronaria y disfunción cardiaca que pueden haber influido en su hiperpotasemia extrema, además del efecto de la acidosis metabólica severa. El bisoprolol, como los otros betabloqueadores adrenérgicos, puede reducir la contractilidad miocárdica en pacientes con disfunción sistólica como este paciente y reducir la frecuencia cardiaca, disminuyendo el débito cardiaco y la perfusión renal, esto ocasiona una caída en la velocidad de filtración glomerular y en la excreción renal de potasio. Adicionalmente los betabloqueadores disminuyen la transformación de prorenina en renina, la actividad de renina plasmática y las concentraciones de angiotensina y aldosterona, reduciendo la capacidad de excreción renal de potasio ${ }^{25}$. La aspirina, al igual que los antiinflamatorios no esteroidales, también puede disminuir la secreción de renina al reducir la producción de prostaglandinas, que fisiológicamente estimulan su secreción ${ }^{26}$. La espironolactona, droga que bloquea los receptores de aldosterona, puede producir hiperpotasemia al dificultar el intercambio de sodio por potasio a nivel de los segmentos distales del túbulo renal, particularmente en los sujetos que reciben simultáneamente betabloqueadores ${ }^{27}$. La interacción de estas drogas puede haber contribuido a alcanzar una concentración extrema del potasio plasmático.

Las arritmias asociadas a hiperpotasemia aparecen más frecuentemente de acuerdo a su magnitud, siendo la fibrilación ventricular o asistolia eventos terminales. No existen reportes en la literatura que definan la concentración letal de la hiperpotasemia, concentraciones de K superiores a $7 \mathrm{mmol} / \mathrm{L}$ son habitualmente mortales ${ }^{28}$. Hasta donde llega nuestro conocimiento, existiría sólo un caso comunicado en la literatura médica de un paciente que sobrevivió a una hiperpotasemia extrema de $14,0 \mathrm{mEq} / \mathrm{L}^{29}$, tal como ocurrió con nuestro paciente. Sin embargo, no estamos en condiciones de argüir las causas por las que nuestro paciente logró sobrevivir una hiperpotasemia extrema que alcanzó $15,4 \mathrm{mEq} / \mathrm{L}$, más allá del tratamiento de urgencia oportunamente instituido.

Consideramos de interés comunicar un caso de hiperpotasemia extrema no fatal, asociada a una acidosis láctica muy probablemente relacionada con sobredosis de metformina, exitosamente corregidas con HDFVVC.

\section{Referencias}

1. United Kingdom Prospective Diabetes Study 24: a 6-year, randomized, controlled trial comparing sulfonylurea, insulin, and metformin therapy in patients with newly diagnosed type 2 diabetes that could not be controlled with diet therapy. United Kingdom Prospective Diabetes Study Group. Ann Intern Med 1998; 128: 165-75.

2. Effect of intensive blood-glucose control with metformin on complications in overweight patients with type 2 diabetes (UKPDS 34). UK Prospective Diabetes Study (UKPDS) Group. Lancet 1998; 352: 854-65.

3. Nathan DM, Buse JB, Davidson MB, Ferrannini E, Holman RR, Sherwin R, et al. Medical management of hyperglycemia in type 2 diabetes: a consensus algorithm for the initiation and adjustment of therapy: a consensus statement of the American Diabetes Association and the European Association for the Study of Diabetes. Diabetologia 2009; 52: 17-30.

4. Bennett WL, Maruthur NM, Singh S, Segal JB, Wilson LM, Chatterjee R, et al. Comparative effectiveness and safety of medications for type 2 diabetes: an update including new drugs and 2-drug combinations. Ann Intern Med 2011; 154: 602-13.

5. Shun C. Phenformin and lactic acidosis: a case report and review. J Emerg Med 1998; 16: 881-6.

6. Bailey CJ. Biguanides and NIDDM. Diabetes Care 1992; 15: 755-72.

7. Aguilar C, Reza A, García JE, Rull JA. Biguanide related lactic acidosis: incidence and risk factors. Arch Med Res 1992; 23: 19-24.

8. Huidobro F, Videla C, Hernández G, Torres H, Jiménez $\mathrm{R}$, Pérez H, et al. Acidosis Láctica en Diabéticos Asociada a Buformina. Rev Med Chile 1989; 117: 46-8.

9. Salpeter SR, Greyber E, Pasternak GA, Salpeter EE. Risk of fatal and nonfatal lactic acidosis with metformin use in type 2 diabetes mellitus: systematic review and metaanalysis. Arch Intern Med 2003; 163: 2594-602.

10. Salpeter SR, Greyber E, Pasternak GA, Salpeter EE. Risk of fatal and nonfatal lactic acidosis with metformin use in type 2 diabetes mellitus. Cochrane Database Syst Rev 2010; 4: CD002967.

11. Tsapas A. Therapeutics. Review: metformin does not increase risk for lactic acidosis in type 2 diabetes. Ann Intern Med 2010; 153: JC1-9.

12. Nyirenda MJ, Sandeep T, Grant I, Price G, McKnight JA. Severe acidosis in patients taking metformin-rapid reversal and survival despite high APACHE score. Diabet Med 2006: 23: 432-5.

13. Wills BK, Bryant SM, Buckley P, Seo B. Can acute overdose of metformin lead to lactic acidosis? Am J Emerg 
Med 2010; 28: 857-61.

14. Rifkin SI, McFarren C, Juvvadi R, Weinstein SS. Prolonged hemodialysis for severe metformin intoxication. Ren Fail 2011; 33: 459-61.

15. Perrone J, Phillips C, Gaieski D. Occult metformin toxicity in three patients with profound lactic acidosis. J Emerg Med 2011; 40: 271-5.

16. Misbin RI, Green L, Stadel BV, Gueriguian JL, Gubbi A, Fleming GA. Lactic acidosis in patients with diabetes treated with metformin. N Engl J Med 1998; 338: 265-6.

17. Lalau JD. Lactic acidosis induced by metformin: incidence, management and prevention. Drug Saf 2010; 33 : 727-40.

18. Stang M, Wysowski DK, Butler-Jones D. Incidence of lactic acidosis in metformin users. Diabetes Care 1999; 22: 925-7.

19. Bailey CJ, Turner RC. Metformin. N Engl J Med 1996; 334: 574-9.

20. Pena JM, Pernaute R, Vicente C. Acute kidney failure and severe lactic acidosis caused by metformin successfully treated with hemodialysis. Nefrologia 2004; 24: 89-90.

21. Panzer U, Kluge S, Kreymann G, Wolf G. Combination of intermittent haemodialysis and high-volume continuous haemofiltration for the treatment of severe metformin-induced lactic acidosis. Nephrol Dial Trans- plant 2004; 19: 2157-8.

22. Mandal AK. Hypokalemia and hyperkalemia. Med Clin North Am 1997; 81: 611-39.

23. Aronson PS, Giebisch G. Effects of pH on Potassium: New Explanations for Old Observations. J Am Soc Nephrol 2011; 22: 1981-89.

24. Adrogué HJ, Madias NE. Changes in plasma potassium concentration during acute acid-base disturbances. Am J Med 1981; 71: 456-67.

25. Blumenfeld JD, Sealey JE, Mann SJ, Bragat A, Marion $\mathrm{R}$, Pecker MS, et al. Beta-adrenergic receptor blockade as a therapeutic approach for suppressing the reninangiotensin-aldosterone system in normotensive and hypertensive subjects. Am J Hypertens 1999; 12: 451-9.

26. Clive DM, Stoff JS. Renal syndromes associated with nonsteroidal antiinflammatory drugs. N Engl J Med 1984; 310: 563-72.

27. Tamirisa KP, Aaronson KD, Koelling TM. Spironolactone-induced renal insufficiency and hyperkalemia in patients with heart failure. Am Heart J 2004; 148: 971-8.

28. Montoliu J, Revert L. Lethal hyperkalemia associated with severe hyperglycemia in diabetic patients with renal failure. Am J Kidney Dis 1985; 5: 47-8.

29. Tran HA. Extreme Hyperkalemia, South Med J 2005; 98 : 729-32. 